The CPD programme for SAMJ is administered by Medical Practice Consulting: CPD questionnaires must be completed online at www.mpconsulting.co.za

True (A) or false (B):

Digitalis reappraised: Still here today, but gone tomorrow?

1. Digoxin is a very complex drug with a very narrow therapeutictoxic window and numerous drug interactions.

2. Digoxin is rapidly absorbed into the circulation, bound to plasma proteins, and largely excreted by the liver and in the stools.

South African National Cancer Registry: Effect of withheld data from private health systems on cancer incidence estimates

3. Regulation 380 of the National Health Act by the Department of Health in 2011 formally established the National Cancer Registry (NCR) as the main cancer surveillance agency, requiring mandatory reporting of all confirmed cancers to the NCR.

Morbidity and mortality of black HIV-positive patients with endstage renal disease (ESRD) receiving chronic haemodialysis

4. Renal disease affects up to $30 \%$ of HIV-infected patients and is associated with increased morbidity and mortality.

5. HIV-associated nephropathy is most common and, unless treated with antiretroviral therapy, progresses rapidly to ESRD.

6. Being HIV-positive is an absolute contraindication to renal transplantation.

7. Because of the high risk of chronic kidney disease (CKD), all HIVpositive patients, even those with high CD4 counts, should be screened for CKD at first encounter with any health service.

The Vaccine and Cervical Cancer Screen (VACCS) project: Linking cervical cancer screening to $\mathrm{HPV}$ vaccination

8. There are 15 HPV high-risk viral types $(16,18,31,33,35,39,45,51,52$, $56,58,59,68,73,82$ ), with the two most oncogenic being HPV 16 and 18.

Human myiasis in rural SA is under-reported

9. Myiasis is infestation of live tissue of humans by larvae (maggots), with ocular myiasis being the most prevalent type.

Multimorbidity in non-communicable diseases (NCDs)

10. The World Health Organization estimates the burden of NCDs to be two to three times higher in SA than in high-income countries.

The unsuspected killer: Liquefied petroleum gas overexposure in South Africa

11. Besides being highly flammable, liquefied petroleum gas can create a hypoxic environment by consuming atmospheric oxygen (especially in small enclosures) to form carbon monoxide, which is toxic; therefore, its use requires adequate ventilation and no leaks.
The utility of urine sulphosalicylic acid testing in the detection of non-albumin proteinuria

12. The bedside detection of non-albumin proteinuria is aided by the use of a simple, inexpensive and often-overlooked investigation: addition of $3 \%$ sulphosalicylic acid to urine.

Digoxin therapy in the modern management of cardiovascular disease: An unusual but serious complication

13. At toxic concentrations, digoxin leads to arrhythmias secondary to increased cell excitability from decreased resting cellular membrane potentials and after depolarisations.

\section{Arthritis mutilans: A rare phenomenon}

14. Psoriatic arthritis is an inflammatory arthropathy associated with psoriasis and usually positive for rheumatoid factor.

For external use only

15. The reported mortality rates for acute hair dye poisoning range between approximately $10 \%$ and $40 \%$.

\section{A tale of two viruses}

16. Herpes zoster in an immunocompromised patient may present with cutaneous and visceral dissemination, the latter in the form of pneumonitis, hepatitis and encephalitis.

Schistosomiasis misdiagnosed as abdominal tuberculosis

17. Schistosomiasis is a waterborne trematode infection that spreads through direct contact with the human skin.

18. The symptoms of schistosomiasis are caused by the host's immune response to the antigens secreted by the schistosoma eggs, leading to a granulomatous reaction.

\section{An additional $X$ chromosome}

19. Most patients with Klinefelter's syndrome do not come to medical attention and therefore do not receive a diagnosis.

Wernicke's encephalopathy as a complication of gastroparesis after emergency partial antrectomy

20. In the clinical presentation of Wernicke's encephalopathy, the typical classic triad of ataxia, confusion and nystagmus is present in less than half of all patients.

CPD questions include articles from CME.

The full versions of each article can be found on the SAMJ website (http://www.samj.org.za)

A maximum of 3 CEUs will be awarded per correctly completed test.

INSTRUCTIONS

1. Read the journal. All the answers will be found there.

2. Go to www.mpconsulting.co.za to answer the questions.

Accreditation number: MDB001/007/01/2014 (Clinical) 